\title{
MicroRNA-125b overexpression in pseudoexfoliation syndrome
}

\author{
Martyna Tomczyk-Socha' ${ }^{1, A-D}$, Dagmara Baczyńska 2,A-C,E,F, Joanna Przeździecka-Dołyk1,C,E, Anna Turno-Kręcicka ${ }^{1, A-C, E, F}$ \\ ${ }^{1}$ Department of Ophthalmology, Wroclaw Medical University, Poland \\ 2 Department of Molecular and Cellular Biology, Faculty of Pharmacy with Division of Laboratory Diagnostics, Wroclaw Medical University, Poland \\ A - research concept and design; $\mathrm{B}$ - collection and/or assembly of data; $\mathrm{C}$ - data analysis and interpretation; \\ $D$ - writing the article; $E$ - critical revision of the article; $F$ - final approval of the article
}

\section{Address for correspondence}

Martyna Tomczyk-Socha

E-mail: martyna_tomczyk@wp.pl

\section{Funding sources}

This study constitutes statutory activity of the Department of Ophthalmology, supported with the Wroclaw Medical University grant No. ST C240.17.014.

\section{Conflict of interest}

None declared

Received on September 11, 2019 Reviewed on February 18,2020 Accepted on June 7, 2020

\begin{abstract}
Background. MicroRNAs (miRs) are small non-coding RNAs. MiR-125b has been described as being downregulated in cataract tissue when compared to a transparent lens.

Objectives. The aims of the study were: 1) to establish the expression of miR-125b in cataracts complicated by pseudoexfoliation syndrome (PEX), glaucoma or PEX glaucoma; and 2) to determine whether any environmental factors influence miR-125b expression.
\end{abstract}

Material and methods. Anterior lens capsules were obtained from 150 patients. The patients were subdivided into 1 of 4 groups: those with PEX (PEXg), those with primary open-angle glaucoma (Gg) and those with PEX glaucoma (PEXGg), plus gender-matched controls with cataracts alone (control group - Cg). Quantitative polymerase chain reaction (qPCR) expression of microRNA-125b was examined in every group.

Results. The mean age of the 150 patients was 75.18 years (standard deviation (SD) \pm 9.12 years). Our investigation indicated, for the first time, that miR-125b expression was increased 3.33 times in the PEXg $(p=0.015)$. The quantitative analysis of miR-125b expression conducted between combined groups of all the patients that have PEX syndrome (with or without glaucoma) and the Cg revealed a statistically significant difference $(p=0.04)$. Lower miR-125b expression was found in the patients who smoked compared to those who did not $(p=0.01)$.

Conclusions. Our data revealed the possible role of miR-125b in PEX syndrome development. There are 2 possible interpretations of these results: either the co-existence of PEX acts as a moderator of miR-125b expression in the anterior lens capsule, or increased expression of miR-125b can play a role in the pathogenesis of PEX.

Key words: microRNA, pseudoexfoliation syndrome, pseudoexfoliation glaucoma, miR-125b, lens capsule

Cite as

Tomczyk-Socha M, Baczyńska D, Przeździecka-Dołyk J,

Turno-Kręcicka A. MicroRNA-125b overexpression in pseudoexfoliation syndrome. Adv Clin Exp Med.

2020;29(12):1399-1405. doi:10.17219/acem/123623

DOI

10.17219/acem/123623

Copyright

Copyright by Author(s)

This is an article distributed under the terms of the

Creative Commons Attribution 3.0 Unported (CC BY 3.0)

(https://creativecommons.org/licenses/by/3.0/) 


\section{Introduction}

MicroRNAs (miRs) are a group of small endogenous noncoding RNAs, generally consisting of 21-23 nucleotides. They regulate posttranscriptional gene expression through complementary or partial complementary binding with the 3 ' untranslated region of target mRNA. ${ }^{1}$ The miRs represent a prominent class of gene regulators. ${ }^{2-4} \mathrm{~A}$ single miR can modulate the expression of several mRNAs, and conversely, a single mRNA strand can interfere with multiple miRs. ${ }^{5}$ Mature miRs have long half-lives, are not rapidly degraded and can influence different biological pathways by binding to different mRNAs. ${ }^{5}$ While miRs usually act as negative modulators of gene expression, they can also stimulate translation. ${ }^{6}$ Previous studies have demonstrated that expression of miRs, such as miR-760, miR-186, miR20a, miR-24, miR-34a, miR-106a, and miR-449a, can be involved in senescence or age-related diseases including neurological disorders, diabetes, degenerative arthritis, and cataracts. ${ }^{2,7}$

Distinct expression profiles of miRs in the central epithelium of transparent human lenses and age-related cataract-affected human lenses have been reported previously. ${ }^{8}$ In transparent lenses, a high expression of miR-184, miR-1826, miR-125b, miR-1308, miR-26a, miR-638, miR923 , and let-7b has been observed. In age-related cataractaffected lenses, other miRs have been identified, including miR-184, miR-1826, let-7b/c, miR-24, mir-23b, miR-23a, and $\mathrm{miR}-923 .{ }^{8}$ It is possible that all the identified miRs are involved in a multifactorial pathogenesis of cataract formation, in which processes of senescence, as well as multiple genetic and environmental factors, have been implicated. Specifically, miR-34a may play an important role in lens senescence, given that its expression in the lens epithelium is positively correlated with age and the severity of the cataract. ${ }^{9}$ There are probably also different exclusively agespecific gene expression changes in human lenses. ${ }^{10}$

The pathogenesis of a cataract is still poorly understood, but Li et al. described lens epithelial cell apoptosis as a common cellular process in cataract development. ${ }^{11}$ Specifically, the TP53 gene is closely associated with lens epithelial cell apoptosis. ${ }^{12}$ Qin et al. described that microRNA-125b (miR-125b) directly targets P53: miR-125b was downregulated in age-related cataract tissue when compared with healthy transparent lens tissue, and inversely related to the level of P53. ${ }^{13}$

With its monolayer structure and direct exposure to ultraviolet radiation, the lens capsule epithelium seems to be an important subject for investigating complex factors playing roles in cellular aging, including genetics and environmental influences. ${ }^{10}$ Senescence has been implicated in cataract, pseudoexfoliation syndrome (PEX) and primary open-angle glaucoma, but disease-specific mechanisms remain elusive.

Pseudoexfoliation syndrome is a common multifactorial age-related and genetically determined elastosis.
Pathologic pseudoexfoliation material (PEXM) accumulates as a result of excessive production and abnormal netting of elastic fibers, resulting in the accumulation of elastic fiber aggregates in tissues of the eye and other organs. ${ }^{14}$

Our study aimed to: 1) establish the expression of miR$125 \mathrm{~b}$ in cataract complicated by other eye diseases; and 2) to determine whether any environmental factors have any influence on miR-125b expression. For the first time we quantified the expression of miR-125b in lens capsules of patients with cataract, PEX, primary open-angle glaucoma, and PEX glaucoma.

\section{Material and methods}

This prospective, controlled study was approved by the Regional Ethics Review Board and was conducted in accordance with the principles set forth in the Guidelines for Good Clinical Practice and the Declaration of Helsinki (and its amendments) (approval No. KB 272/2017 from 26/04/2017).

Fresh specimens of anterior lens capsules were obtained from 150 patients between 46 and 92 years of age (mean: 75.18 years, standard deviation (SD) \pm 9.12 years) during cataract phacoemulsification surgery. All lens samples were obtained with intact continuous curvilinear capsulorhexis 5-6 $\mathrm{mm}$ in diameter.

Each patient underwent a complete ophthalmological examination comprising an interview, anterior segment slit lamp biomicroscopy before and after dilation of the pupil, intraocular pressure (IOP) and funduscopic examination. Patients who had previously undergone eye surgery or who had other ophthalmic diseases (e.g., diabetic retinopathy, uveitis, trauma) were excluded.

The patients were consecutively selected and assigned to 1 of 4 groups:

1. group of patients with cataract and PEX (PEXg) -38 patients;

2. group of patients with cataract and primary openangle glaucoma $(\mathrm{Gg})$ - 37 patients;

3. group of patients with cataract, PEX and glaucoma (PEX glaucoma group - PEXGg) - 30 patients;

4. group of patients with cataract - the control group (Cg) -45 patients.

The severity of the cataracts was similar in each study group. Information regarding current ophthalmic medication usage (timolol, brymonidine, prostaglandin analog, dorzolamide) and lifestyle data, such as smoking habits, were elicited through a patient interview prior to surgery.

\section{RNA isolation and relative real-time RT-PCR}

Fragments of lens capsules from patients with cataract were homogenized using MagNA Lyser Green Beads (Roche Diagnostics GmbH, Mannheim, Germany) in a lysis 
buffer. Total RNA was isolated using a NucleoSpin RNA II kit (Macherey-Nagel \& Co., Düren, Germany) following the manufacturer's protocol. Reverse transcription reaction (RT) was performed using $3 \mu \mathrm{L}$ of extracted total RNA, TaqMan MicroRNA Reverse Transcription Kit (Thermo Fisher Scientific, Foster City, USA) and specific RT primers for RNU6B (Thermo Fisher Scientific) and miR-125b (Thermo Fisher Scientific) in a final volume of $15 \mu \mathrm{L}$ according to the manufacturer's instructions. Kapa PROBE Master Mix (Kapa Biosystems Inc., Boston, USA) and specific TaqMan MicroRNA Assays (Thermo Fisher Scientific) were used to assess microRNA expression according to the manufacturers' instructions. We added $1.33 \mu \mathrm{L}$ of twice-diluted RT product to single real-time polymerase chain reaction (RT-PCR). All the reactions were performed in triplicate in 96-well plates under the following thermal cycling conditions: $20 \mathrm{~s}$ at $95^{\circ} \mathrm{C}$ followed by 40 cycles of $1 \mathrm{~s}$ at $95^{\circ} \mathrm{C}$ and $20 \mathrm{~s}$ at $60^{\circ} \mathrm{C}$. The reactions were run in the 7900 HT Fast Real-Time PCR System (Thermo Fisher Scientific) and the threshold cycle data $(\mathrm{Ct})$ were collected using SDS v. 2.2 software (Thermo Fisher Scientific). The relative expression of miR-125b in each sample was calculated using the comparative $C t$ method $\left(2^{-\Delta \Delta C t}\right)$. The patients' samples were normalized to RNU6B, calibrated to the $\mathrm{Cg}$ and presented as a relative quantity (RQ).

\section{Statistical analysis}

A qualitative analysis ( $\mathrm{X}^{2}$ test) used to form a categorized table and a quantitative analysis (Student's t-test) were performed. Analysis of variance (ANOVA) with a post hoc least significant difference (LSD) evaluation was used to define the differences between the groups. A partial analysis was performed at intervals of RQ (and $\operatorname{logRQ}$ ) defined with the $X^{2}$ method. In addition, the correlation between miR-125b and the age of the patients was assessed. Logistic regression was used to assess the probability of influence of the RQ in each specified group. The results were considered statistically significant at a p-value less than 0.05. All the calculations were carried out using STATISTICA v. 12 software. (StatSoft Inc., Tulsa, USA) and MedCalc (MedCalc Software Ltd., Ostend, Belgium).

\section{Results}

\section{MiR-125b expression}

In order to determine the expression level of miR-125b in every group, relative RT-PCR was performed. The level of miR-125b expression was increased in each of the study groups (PEXg, Gg and PEXGg) compared to the Cg (Fig. 1). The qualitative analysis ( $X^{2}$ test) revealed statistically significant increases in the miR-125b levels in the PEXg cohort compared to the $\mathrm{Cg}$ cohort (3.33 times higher; $\mathrm{p}=0.015$, Table 1 ). There were no statistically significant differences in miR-125b levels between the samples from the $\mathrm{Gg}$ and $\mathrm{Cg}$ cohorts.

Table 1 shows the mean and standard deviations of RQ values; however, the RQ distributions are right-skewed. Therefore, the ANOVA test was used after the RQ logarithm transformation. An LSD test was used as a as post hoc test after the ANOVA. No differences in sex distribution between groups was found ( $\mathrm{p}=0.4822)$. In the PEXg compared to the other 2 groups (Gg and PEXGg), the number of patients with an RQ value $>0.6$ was increased by $10 \%$, while $10 \%$ fewer patients had an RQ value $<-0.3$. This further supports the statistical difference between the groups described (Fig. 2).

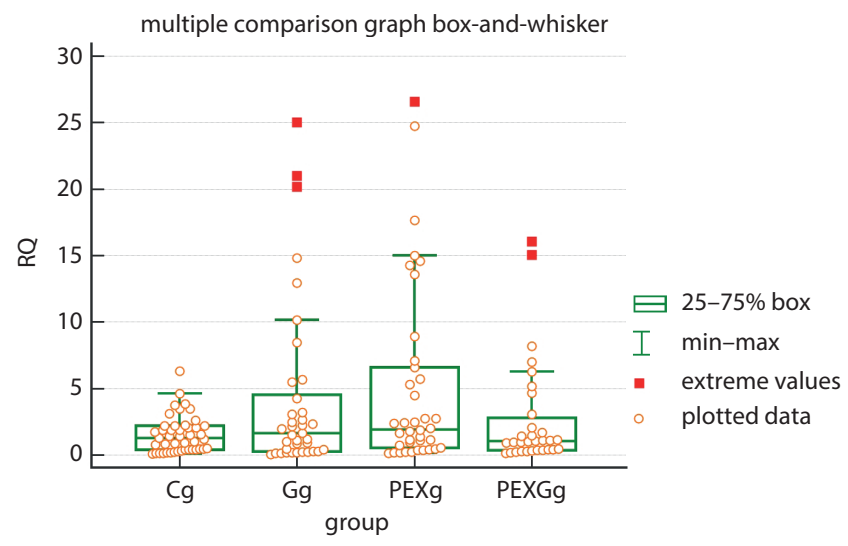

Fig. 1. Comparison of miR-125b expression levels in PEXg, Gg, PEXGg, and $\mathrm{Cg}$. The observed values were higher in the study groups with an ophthalmic disease compared to the Cg, but the only statistically significant difference was in PEXg compared to $\mathrm{Cg}$

$p=0.046$ (median - horizontal line inside of the $25-75 \%$ box).

Table 1. Mean expression of miR-125b in every group (LSD test as post hoc test)

\begin{tabular}{|c|c|c|c|c|c|c|}
\hline Group & $\begin{array}{l}\text { Number } \\
\text { of patients }\end{array}$ & Mean age ${ }^{\dagger}$ & $\begin{array}{l}\text { Sex proportion in each } \\
\text { group (female:male) }{ }^{\dagger}\end{array}$ & Mean RQ & SD & $\begin{array}{c}\text { p-value } \\
\text { (tested group vs Cg) }\end{array}$ \\
\hline PEXg & 38 & 77 & $15: 4$ & 5.11 & 6.92 & 0.015 \\
\hline $\mathrm{Gg}$ & 37 & 72 & $25: 12$ & 4.30 & 6.44 & 0.14 \\
\hline PEXGg & 30 & 76 & $22: 8$ & 2.78 & 4.10 & 0.45 \\
\hline $\mathrm{Cg}$ & 45 & 73 & $29: 16$ & 1.53 & 1.38 & $x$ \\
\hline All groups & 150 & 75 & $53: 22$ & 3.37 & 5.27 & $x$ \\
\hline
\end{tabular}

${ }^{+}$no statistically significant differences between groups ( $p>0.05$ ); LSD - least significant difference; SD - standard deviation; RQ - relative quantity; PEX - pseudoexfoliation; PEXg - patients cataract and PEX; PEXGg - patients with cataract, glaucoma and PEX; Gg - patients with cataract and primary open-angle glaucoma; $\mathrm{Cg}$ - control group. 


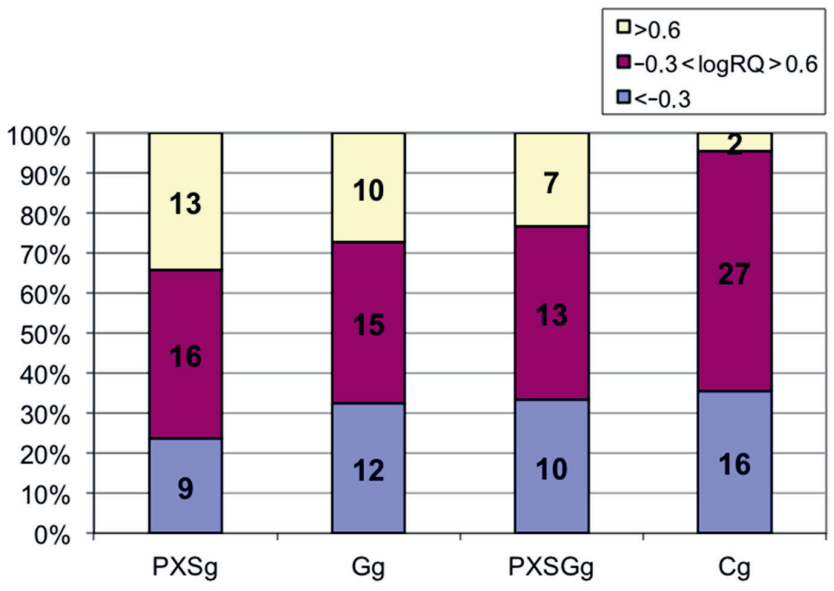

Fig. 2. MiR-125b expression analysis in PEXg, Gg, PEXGg, and Cg with subdivisions into the different value ranges of logRQ (the number of patients in each group is on the bar)

The quantitative analysis of miR-125b expression (Student's t-test) conducted between PEXg+PEXGg (the combined group of all the patients that have the PEX syndrome,

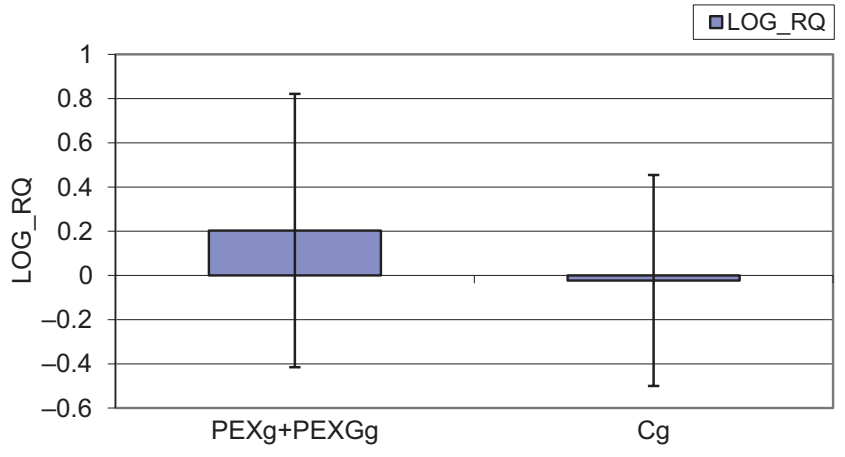

Fig. 3. MiR-125b expression (logRQ values) in all the patients with PEX syndrome compared to the $\mathrm{Cg}$ (mean logRQ PEXg+PEXGg $=0.203$; $\mathrm{Cg}=-0.0228 ; \mathrm{p}=0.04)$

with or without glaucoma) and Cg revealed a statistically significant difference ( $\mathrm{p}=0.04$, Fig. 3).

Additionally, the logistic regression model (Fig. 4) revealed a statistically significant correlation (probability $>50 \%$ ) between the level of RQ and the diagnoses outlined in the study: A) existing PEX with or without glaucoma;
A

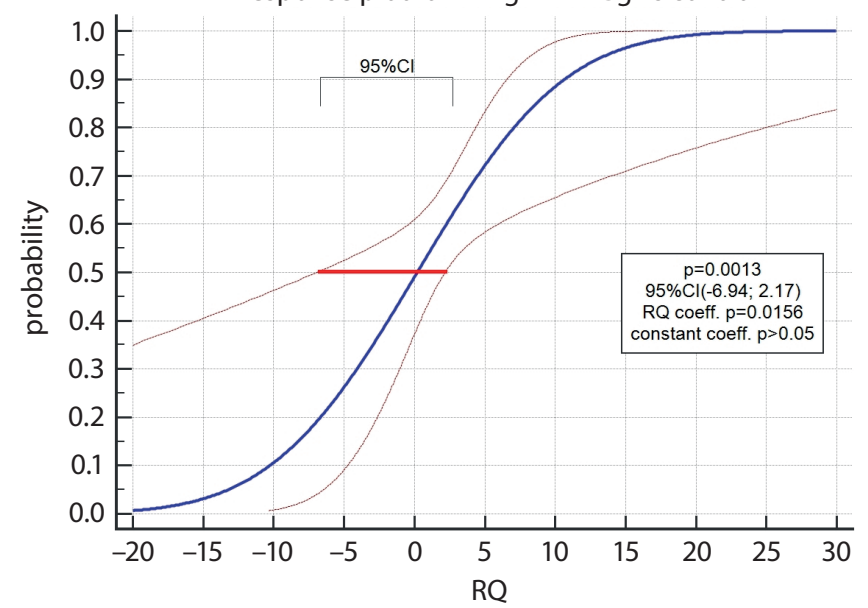

C

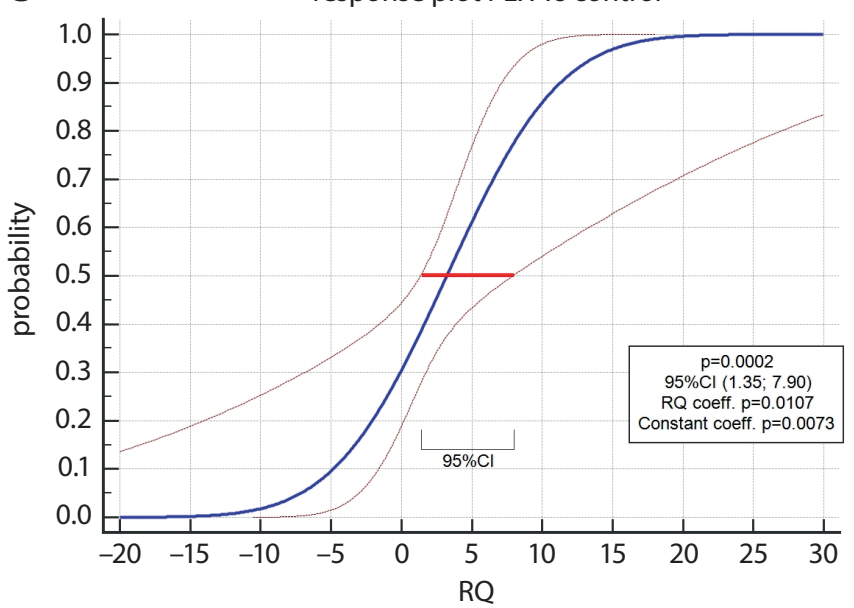

B

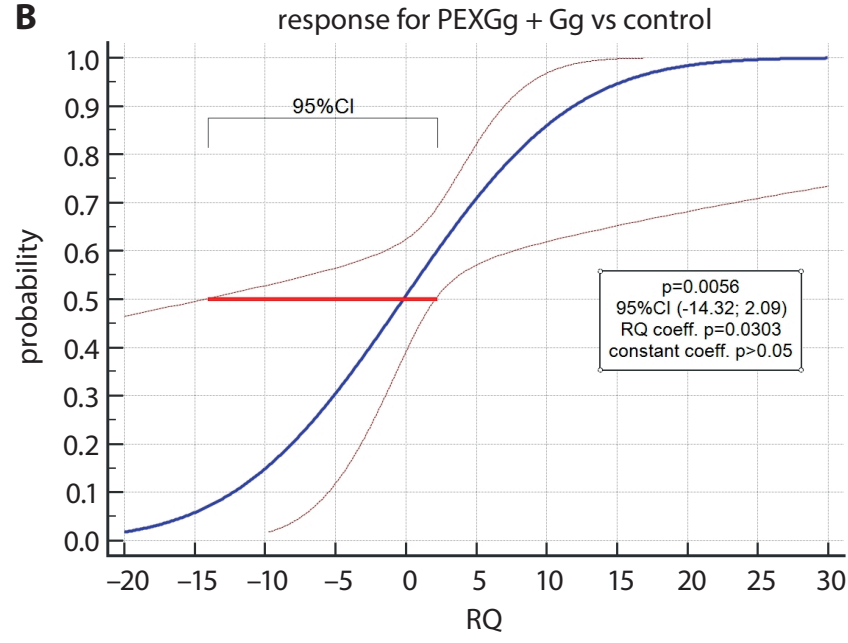

D

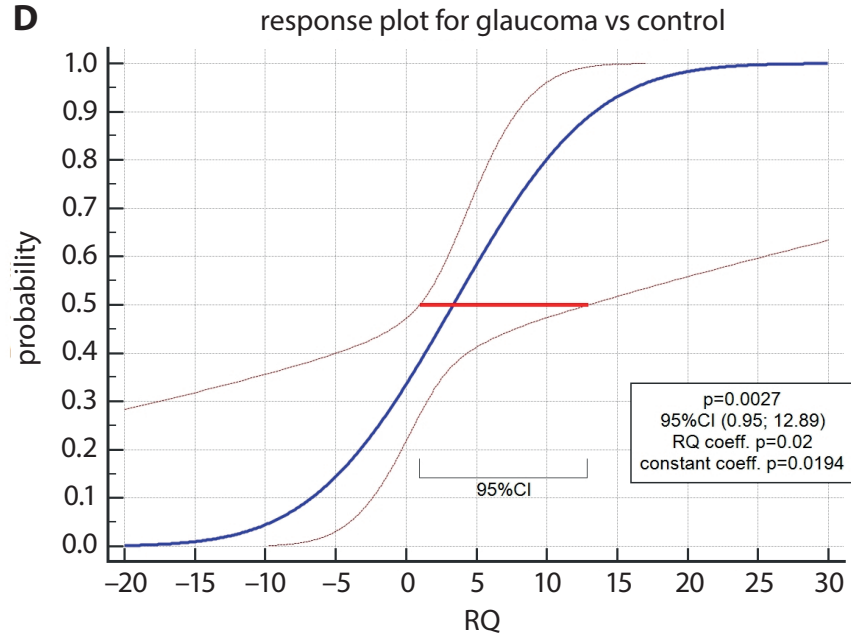

Fig. 4. Logistic regression analysis for different categorical outcomes (A) existing PEX with or without glaucoma, (B) existing glaucoma with or without PEX, (C) only PEX, and (D) only glaucoma compared to the Cg. The PEXGg is the factor that reverses the $95 \% \mathrm{Cl} R \mathrm{RQ}$ values from predominantly positive in the PEXg and Gg into the predominantly negative in the combined groups such as PEXg+PEXGg and PEXGg+Gg 
B) existing glaucoma with or without PEX, C) only PEX; and D) only glaucoma compared to the Cg. The PEXG is a factor that reverses the 95\% confidence interval (95\%) CI RQ values from predominantly positive in the PEXg and Gg into the predominantly negative in the combined groups, such as PEXg+PEXGg and PEXGg+Gg. The p-values and 95\% CI ranges are: $0.0013,-6.94-2.17 ; 0.0056,-14.32-2.09$; 0.0002, 1.35-7.90; and 0.0027, 0.95-12.89, respectively, for PEXg+PEXGg, PEXGg+Gg, PEXg, and Gg.

\section{Factors that may affect miR-125b expression}

There were no statistically significant correlations between the levels of miR-125b and the age of the patients. During the assessment of relationships between levels of miR-125b and important factors obtained from patients' history records (current cigarettes smoking, drops for glaucoma), only 1 statistically significant difference was found: a lower level of miR-125b expression was found in patients who smoked compared to those who did not $(\mathrm{p}=0.01$, Table 2). Cigarette smoking was noted in the histories of $5.26 \%$ of the patients with PEXg ( 2 of 38 patients), $16.67 \%$ of the patients with PEXGg (5 of 30 patients), $8.1 \%$ of the patients with Gg (3 of 37 patients), and $8.89 \%$ of the patients in the $\mathrm{Cg}$ ( 4 of 45 patients).

\section{Discussion}

Our study is the first to present the correlation between the level of miR-125b and PEX syndrome in patients with cataracts and PEX compared to patients with cataracts alone.

MiR-125b has been demonstrated to be inversely related to the level of P53 in age-related cataract tissue and is involved in aging induced by the DNA damage response, which is mainly governed by the P53 pathway. ${ }^{13}$ Some confirmed risk factors for cataract formation, like chronic ultraviolet light exposure and oxidative stress, cause DNA damage as a result of the cumulative effects of reactive oxygen species (ROS). ${ }^{9}$

In our study, we observed no differences in the levels of expression of miR-125b in the lens capsules of patients with cataracts alone and those with both cataract and primary open-angle glaucoma. The main finding of our study is that miR-125b is significantly increased in lens capsules of patients with PEXg. The results obtained in this and in prior studies allow to us assume that in the examined population, the co-existence of PEX and cataracts was the main moderator of miR-125b expression in the lens capsules and that the increased expression of miR-125b may be a cause of PEX. It should be emphasized that our research, which reflects miR-125b expression levels close to those seen in vivo, was carried out on a large number of sampled capsules from a patient population that was relatively homogenous in terms of age and disease severity.

Numerous mechanisms, such as disruptions in the proteolytic balance of the extracellular matrix, chronic inflammatory processes of low intensity, chronic or recurrent ischemia/hypoxia, regulation disturbances at the cellular level, and oxidative stress, have all been implicated in the formation of PEXM complexes. ${ }^{4,15,16}$ These factors interplay in creating conditions specific for PEX and the upregulation of miR-125b in capsular lens tissue can be either a cause or an effect of these processes.

Altered expression of miR-125b has been demonstrated in a number of chronic diseases and malignancies, including prostate, breast and pancreatic cancer and primary hepatocellular carcinoma. ${ }^{17-20} \mathrm{MiR}-125 \mathrm{~b}$ expression is associated with classic phenotypic and genotypic sequelae of reduced P53 expression, including altered sensitivity to genotoxic agents and reduced expression of P53-related transcriptional targets such as miR-34a/b/c. ${ }^{21}$

Excessive oxidative stress has been shown to augment expression of miR-125b. ${ }^{21}$ Upregulation of miR-125b expression has been identified in acute cerebral ischemia. ${ }^{22,23}$ Also, miR-125b has been shown to increase in settings

Table 2. Summary of additional factors in the analysis of miR-125b expression

\begin{tabular}{|c|c|c|c|c|c|c|c|c|}
\hline \multirow{2}{*}{\multicolumn{2}{|c|}{ Medical history information }} & \multicolumn{2}{|c|}{ Mean } & \multicolumn{2}{|c|}{ Number of valid cases } & \multicolumn{2}{|c|}{ SD } & \multirow{2}{*}{$\mathrm{p}$-value } \\
\hline & & no & yes & no & yes & no & yes & \\
\hline \multirow{3}{*}{ Medications } & timololi maleas & 1.91 & 1.84 & 131 & 19 & 0.72 & 0.76 & 0.57 \\
\hline & brimonidine & 1.90 & 2.00 & 131 & 19 & 0.70 & 0.88 & 0.96 \\
\hline & prostaglandins & 1.89 & 2.00 & 137 & 13 & 0.72 & 0.71 & 0.77 \\
\hline Habits & smoking & 1.95 & 1.43 & 136 & 14 & 0.72 & 0.51 & 0.01 \\
\hline \multirow{5}{*}{ Comorbidities } & $\mathrm{DM}$ & 1.88 & 1.97 & 119 & 31 & 0.72 & 0.75 & 0.52 \\
\hline & heart failure & 1.87 & 1.95 & 91 & 59 & 0.70 & 0.75 & 0.95 \\
\hline & $\mathrm{HT}$ & 1.88 & 1.91 & 52 & 98 & 0.70 & 0.73 & 0.90 \\
\hline & dementia & 1.90 & 1.80 & 145 & 5 & 0.73 & 0.45 & 0.69 \\
\hline & vertigo & 1.91 & 1.75 & 138 & 12 & 0.72 & 0.75 & 0.60 \\
\hline
\end{tabular}

DM - diabetes mellitus; HT - hypertension; SD - standard deviation. 
of hypoxia. ${ }^{24}$ Thus, oxidative stress and hypoxia appear to play a major role in the modification of gene expression.

The impact of smoking on the incidence of PEX has not been clearly assessed, but Spečkauskas et al. reported that the age-adjusted odds ratio (OR) for PEX increased 3.1\% with each year of smoking, but that the number of cigarettes smoked per day had no effect on PEX. ${ }^{25}$ However, other researchers, like You et al., found no relationship between smoking and the occurrence of PEX in a large cohort study. ${ }^{26}$

The present study showed significantly reduced miR$125 \mathrm{~b}$ expression in cigarette smokers. Analyzing the distribution of smokers among the study groups, the percentage of smoking patients in the PEXGg was close to double that in the other groups (16.67\% compared to $5.26 \%$ in PEXg, 8.1\% in $\mathrm{Gg}$ and $8.89 \%$ in $\mathrm{Cg}$ ).

In the present study, there was no significant elevation in the expression of miR-125b in PEXGg. Increased expression was statistically significant for PEXg and for the combined group of all patients with PEX (PEXg+PEXGg). If smoking reduces the expression level of miR-125b, and more patients who smoke cigarettes are found in PEXGg than in the other groups, it can be assumed that the reason for the absence of a statistically significant increase in miR-125b expression in the PEXGg may be the increased percentage of smokers. In the group of all patients with PEX, the percentage of smoking patients was equalized (10.29\% in the PEXg+PEXGg group compared to $8.89 \%$ in the $\mathrm{Cg}$ ) and significantly increased miR-125b expression was noted in all the PEX patients.

The central fragment of the anterior lens capsule was the material examined to assess the miR-125b level. The lens capsule is an ideal source of information about PEX, as it is constantly exposed to damaging factors that participate in the disease pathogenesis, such as UV rays. It is a region in which the molecules comprising PEXM are deposited and bound in insoluble structures. The lens capsule is avascular and is washed by aqueous fluid. The components of the aqueous fluid can impact the lens capsule. According to the protein sink model, the anterior chamber of PEX eyes reflects a breakdown of the bloodeye barrier. ${ }^{14}$ Chronic inflammatory changes of the aqueous humor may lead to dysregulation of miR expression and influence P53 activity, leading to the transcription of numerous genes responsible for senescence or apoptosis by activation of the target mRNA. ${ }^{27}$

\section{Conclusions}

An interesting and innovative aspect of our preliminary study was the correlation of PEX and miR-125b expression in the eye tissue examined. Further studies are needed to determine the role of miR in eye pathologies. Dysregulation of miR expression and activity can contribute to stress-related chronic diseases such as PEX. Even with the advances in the field in the last decade, effective novel therapeutic approaches are still lacking for PEX. An improved understanding of the impact of epigenetic factors, such as DNA hypermethylation and histone modifications in the regulation of miR expression, is needed. The results of this study suggest that miR shows a potential to be a future avenue for effective diagnosis and therapeutic strategies for PEX.

\section{ORCID iDs}

Martyna Tomczyk-Socha (D) https://orcid.org/0000-0002-1472-4996 Dagmara Baczyńska (D) https://orcid.org/0000-0001-6781-6758 Joanna Przeździecka-Dołyk (D) https://orcid.org/0000-0002-1099-4876 Anna Turno-Kręcicka (D) https://orcid.org/0000-0001-6732-1851

\section{References}

1. Dogini DB, Pascoal VD, Avansini SH, Vieira AS, Pereira TC, LopesCendes I. The new world of RNAs. Genet Mol Biol. 2014;37(1 Suppl): 285-293.

2. Lee YH, Kim SY, Bae YS. Upregulation of miR-760 and miR-186 is associated with replicative senescence in human lung fibroblast cells. Mol Cells. 2014;37(8):620-627.

3. Chen K, Rajewsky N. The evolution of gene regulation by transcription factors and microRNAs. Nat Rev Genet. 2007;8(2):93-103.

4. Peng $\mathrm{CH}$, Liu JH, Woung LC, et al. MicroRNAs and cataracts: Correlation among let-7 expression, age and severity of lens opacity. Br J Ophthalmol. 2012;96(5):747-751.

5. Kowlru RA, Kowlru A, Mishra M, Kumar B. Oxidative stress and epigenetic modifications in the pathogenesis of diabetic retinopathy. Prog Retin Eye Res. 2015;48:40-61.

6. Vasudevan S, Tong Y, Steitz JA. Switching from repression to activation: MicroRNAs can up-regulate translation. Science. 2007;318(5858): 1931-1934.

7. Khee SG, Yusof YA, Makpol S. Expression of senescence-associated microRNAs and target genes in cellular aging and modulation by tocotrienol-rich fraction. Oxid Med Cell Longev. 2014:725929. doi:10. $1155 / 2014 / 725929$

8. Wu C, Lin H, Wang Q, et al. Discrepant expression of microRNAs in transparent and cataractous human lenses. Invest Ophthalmol Vis Sci. 2012;53(7):3906-3912.

9. Chien KH, Chen SJ, Liu JH, Chang HM, Woung LC, Liang CM. Correlation between microRNA-34a levels and lens opacity severity in agerelated cataracts. Eye (Lond). 2013;27(7):883-888.

10. Hawse JR, Hejtmancik JF, Horwitz J, Kantorow M. Identification and functional clustering of global gene expression differences between age-related cataract and clear human lenses and aged human lenses. Exp Eye Res. 2004;79(6):935-940.

11. Li WC, Kuszak JR, Dunn K, et al. Lens epithelial cell apoptosis appears to be a common cellular basis for non-congenital cataract development in humans and animals. J Cell Biol. 1995;130(1):169-181.

12. Geatrell JC, Gan PM, Mansergh FC, et al. Apoptosis gene profiling reveals spatio-temporal regulated expression of the $\mathrm{p} 53 / \mathrm{Mdm} 2$ pathway during lens development. Exp Eye Res. 2009;88(6):1137-1151.

13. Qin Y, Zhao J, Min X, et al. MicroRNA-125b inhibits lens epithelial cell apoptosis by targeting p53 in age-related cataract. Biochim Biophys Acta. 2014;1842(12 Pt A):2439-2447.

14. Lee RK. The molecular pathophysiology of pseudoexfoliation glaucoma. Curr Opin Ophthalmol. 2008;19(2):95-101.

15. Wu C, Liu Z, Ma L, et al. MiRNAs regulate oxidative stress related genes via binding to the $3^{\prime}$ UTR and TATA-box regions: A new hypothesis for cataract pathogenesis. BMC Ophthalmol. 2017;17(1):142.

16. Zenkel M, Kruse FE, Naumann GO, Schlötzer-Schrehard U. Impaired cytoprotective mechanisms in eyes with pseudoexfoliation syndrome/glaucoma. Invest Ophthalmol Vis Sci. 2007;48(12):5558-5566.

17. Wojciechowska A, Braniewska A, Kozar-Kamińska K. MicroRNA in cardiovascular biology and disease. Adv Clin Exp Med. 2017;26(5):865-874.

18. Shi XB, Xue L, Yang J, et al. An androgen-regulated miRNA suppresses Bak1 expression and induces androgen-independent growth of prostate cancer cells. Proc Natl Acad Sci U S A. 2007;104(50):19983-19988. 
19. Thomson JM, Newman M, Parker JS, Morin-Kensicki EM, Wright T, Hammond SM. Extensive post-transcriptional regulation of microRNAs and its implications for cancer. Genes Dev. 2006;20(16):2202-2207.

20. Xu J, Li J, Zheng TH, Bai L, Liu ZJ. MicroRNAs in the occurrence and development of primary hepatocellular carcinoma. Adv Clin Exp Med. 2016;25(5):971-975.

21. SelbachM,SchwanhäusserB,ThierfelderN,FangZ,KhaninR, RajewskyN. Widespread changes in protein synthesis induced by microRNAs. Nature. 2008;455(7209):58-63.

22. Lukiw WJ, Pogue Al. Induction of specific microRNA (miRNA) species by ROS-generating metal sulfates in primary human brain cells. J Inorg Biochem. 2007;101(9):1265-1269.
23. Sepramaniam S, Tan JR, Tan KS, et al. Circulating microRNAs as biomarkers of acute stroke. Int J Mol Sci. 2014;15(1):1418-1432.

24. Kulshreshtha R, Ferracin M, Wojcik SE, et al. A microRNA signature of hypoxia. Mol Cell Biol. 2006;27(5):1859-1867.

25. Spečkauskas $M$, Tamošiūnas $A$, Jašinskas V. Association of ocular pseudoexfoliation syndrome with ischaemic heart disease, arterial hypertension and diabetes mellitus. Acta Ophthalmol. 2012;90(6): e470-e475.

26. You QS, Xu L, Wang YX, et al. Pseudoexfoliation: Normative data and associations - the Beijing eye study 2011. Ophthalmology. 2013;120(8): 1551-1558.

27. Liu B, Chen Y, Clair DK. ROS and p53: A versatile partnership. Free Radic Biol Med. 2008;44(8):1529-1535. 